\title{
STEMI due to septic embolism from prosthetic aortic valve Aspergillus spp. Endocarditis in an immunocompetent patient.
}

\author{
coral Serrano Arroyo ${ }^{1}$, Luis Sánchez Trujillo², Alan León Bojórquez², José Leal Franco², \\ Amalia Castro Rodríguez ${ }^{2}$, and Rosa Mejía Bañuelos ${ }^{2}$ \\ ${ }^{1}$ Mexican Social Security Institute \\ ${ }^{2}$ Instituto Mexicano del Seguro Social
}

May 24, 2021

\begin{abstract}
A 65-year-old man with a history of aortic valve replacement surgery and non-coronary artery disease was admitted to the emergency department with acute ST-segment elevation myocardial infarction (STEMI). Coronary angiography revealed left anterior descending (LAD) artery occlusion, which was treated successfully with angioplasty. The patient developed obstructive valvular dysfunction, and large vegetation was observed on echocardiography. The patient subsequently developed irreversible septic shock and died several days later. The pathology report identified Aspergillus as the etiologic agent.
\end{abstract}

STEMI due to septic embolism from prosthetic aortic valve Aspergillus spp. Endocarditis in an immunocompetent patient.

Affiliations: MD Coral Serrano Arroyo ${ }^{1}$, MD Luis A. Sánchez-Trujillo ${ }^{1,}$ MD Alan A. León Bojórquez ${ }^{1}$, MD José A. Leal Franco ${ }^{2}$, MD Amalia Castro Rodríguez ${ }^{3}$, MD Rosa M. Mejía Bañuelos ${ }^{3} 1$ Cardiology Department, 2 Vascular Surgery Department, 3 Pathology Department at Instituto Mexicano Del Seguro Social, UMAE 34 Hospital de Cardiología. Monterrey, México.

\section{Abstract}

A 65-year-old man with a history of aortic valve replacement surgery and non-coronary artery disease was admitted to the emergency department with acute ST-segment elevation myocardial infarction (STEMI). Coronary angiography revealed left anterior descending (LAD) artery occlusion, which was treated successfully with angioplasty. The patient developed obstructive valvular dysfunction, and large vegetation was observed on echocardiography. The patient subsequently developed irreversible septic shock and died several days later. The pathology report identified Aspergillus as the etiologic agent.

\section{Case}

We report the case of a 65-year-old man with a history of severe aortic stenosis and aortic prosthetic valve replacement. Pre-surgical coronary angiography showed no significant obstructions, and other comorbidities were discarded. The patient recovered without complications and was discharged home with anticoagulant treatment. One month after surgery, he arrived at the emergency department complaining of sudden oppressive retrosternal chest pain and dyspnea. On physical examination, his heart rate was 74 beats per minute, he was found to be diaphoretic, hypotensive, and tachypneic with $96 \%$ oxygen saturation. Thoracic examination revealed no rales and prosthetic valve sounds without murmurs. His leukocyte, glucose, troponin, and B-type natriuretic peptide (BNP) levels were $8.5 \times 109 / \mathrm{L}, 143 \mathrm{~g} / \mathrm{dL}, 120 \mathrm{ng} / \mathrm{ml}$ and $659 \mathrm{pg} / \mathrm{mL}$, respectively. The initial electrocardiogram showed anterolateral ST-segment elevation myocardial infarction (STEMI). Emergency 
coronariography was performed, revealing proximal left anterior descending (LAD) artery embolic occlusion, and coronary balloon angioplasty was successfully performed. (Figure 1)

During recovery, the patient presented with fever; blood cultures were taken, and empirical vancomycin was initiated. Transthoracic echocardiography (TTE) showed normal prosthetic function and no vegetation. Blood cultures were negative, and clinical evolution was satisfactory, so he was discharged home. The patient was readmitted to the emergency department three weeks later with fever, cardiogenic shock, and complete atrioventricular block, so a temporal pacemaker was placed. Upon physical examination, a new aortic systolic murmur was auscultated without prosthetic click sounds. Urgent TTE revealed 14 x $15 \mathrm{~mm}$ vegetation at the prosthetic aortic valve. Septic and cardiogenic shock were diagnosed and attributed to infective endocarditis. The patient experienced cardiac arrest and died a few hours later, despite aggressive management. The postmortem pathology report confirmed Aspergillus ssp , prosthetic aortic valve endocarditis, and anterolateral myocardial infarction. (Figure 2)

\section{Learning objectives}

- Identify clinical presentation and risk factors for the embolic coronary acute syndrome as the onset of Aspergillus Endocarditis (AE).

- Review of the diagnosis and treatment of AE and septic embolic STEMI.

\section{Discussion}

Fungal endocarditis (FE) is an extremely rare cause of septic embolism, especially in immunocompetent patients. AE is reported in $20-30 \%$ of all $\mathrm{FE}$ cases, affecting nearly $0.1 \%$ of all prosthetic cardiac valves. Congenital heart disease, prosthetic valves, malignancy, solid-organ transplant, bone marrow transplant, and immunosuppression are the main risk factors for FE. ${ }^{1}$

The diagnosis of $\mathrm{FE}$ is challenging because the clinical symptoms are non-specific. In nearly $50 \%$ of cases, the diagnosis is postmortem. Blood cultures are often negative, even in disseminated infection. Some nonculture-based diagnostic methods, such as galactomannan enzyme immunoassay, are approved by the United States Food and Drug Administration (FDA). However, histological confirmation remains the gold standard for diagnosis. ${ }^{2}$

The clinical presentation of $\mathrm{AE}$ is vague, with fever and cardiac murmurs being the most common features, followed by embolization-related symptoms and cardiac failure. Clinically significant coronary embolism (CE) has been reported in $1.5 \%$ of cases with infective endocarditis, micro-emboli to the coronary arteries were present in more than $60 \%$ of cases on postmortem examination, particularly in mitral valve IE, vegetation larger than $10 \mathrm{~mm}$, and fungal endocarditis. ${ }^{3} \mathrm{CE}$ is considered definite in the following scenarios: angiographic evidence of $\mathrm{CE}$ and thrombosis without arteriosclerotic lesions, $\mathrm{CE}$ in several locations simultaneously, or the presence of concomitant systemic embolization not attributable to an apical thrombus. ${ }^{4}$

In this case, we performed a balloon angioplasty with aspiration thrombectomy which is the preferred treatment in STEMI secondary to septic embolism. Experience with thrombolytic therapy has been unfavorable and associated with a high risk of complications and low efficacy. Cardiac complications of septic coronary embolization during percutaneous transluminal coronary angioplasty are mycotic septic aneurysm, perforation, carditis, and resistant vegetations. ${ }^{4}$ We empirically initiated Vancomycin. Nevertheless, Voriconazole is the antifungal therapy of choice; some experts recommend the addition of an echinocandin or amphotericin B. ${ }^{5}$

\section{Conclusion}

STEMI due to septic CE is a rare complication of IE and has a high mortality. Diagnosing fungal endocarditis requires a high clinical index of suspicion, given the initial non-specific presentation. A large vegetation observed in the TTE is highly suggestive of fungal endocarditis. STEMI treatment in the presence of septic $\mathrm{CE}$ is still controversial and should be modified individually. 


\section{Reference:}

1. Penelope D Barnes, et al. Aspergillosis: spectrum of disease, diagnosis, and treatment. Infect Dis Clin North Am 2006; 20:545-61.

2. Habib G, et al. 2015 ESC Guidelines for the management of infective endocarditis. Eur Heart J. 2015; 36: 3075-123. https://doi.org/10.1093/ eurheartj/ehv319 PMID: 26320109

3. Kalokhe, et al. Aspergillus endocarditis: a review of the literature. International Journal of Infectious Diseases 14 (2010) e1040-e1047.

4. Shibata $\mathrm{T}$ et al. Prevalence, Clinical Features and Prognosis of Acute Myocardial Infraction Attributable to coronary Artery Embolism. Ciruculation 2015; 132 (4): 241-250.

5. Claire E. Raphael, et al. Coronary Embolus. J Am Coll Cardiol Intv. 2018 January, 11 (2) 172-180.

Corresponding author: MD Coral Serrano Arroyo. Cardiology Department at UMAE Hospital de Cardiología No. 34, Mexican Institute of Social Security, Monterrey, Nuevo León México.

E-mail for correspondence: coral.se.ar@gmail.com, phone number: +52 8110049952. 